\title{
ETHNOPRED: a novel machine learning method for accurate continental and sub-continental ancestry identification and population stratification correction
}

Mohsen Hajiloo ${ }^{1,2}$, Yadav Sapkota ${ }^{3,5}$, John R Mackey ${ }^{4,5}$, Paula Robson ${ }^{5,6}$, Russell Greiner ${ }^{1,2+}$ and Sambasivarao Damaraju $u^{3,5^{*}+}$

\begin{abstract}
Background: Population stratification is a systematic difference in allele frequencies between subpopulations. This can lead to spurious association findings in the case-control genome wide association studies (GWASs) used to identify single nucleotide polymorphisms (SNPs) associated with disease-linked phenotypes. Methods such as selfdeclared ancestry, ancestry informative markers, genomic control, structured association, and principal component analysis are used to assess and correct population stratification but each has limitations. We provide an alternative technique to address population stratification.

Results: We propose a novel machine learning method, ETHNOPRED, which uses the genotype and ethnicity data from the HapMap project to learn ensembles of disjoint decision trees, capable of accurately predicting an individual's continental and sub-continental ancestry. To predict an individual's continental ancestry, ETHNOPRED produced an ensemble of 3 decision trees involving a total of 10 SNPs, with 10-fold cross validation accuracy of 100\% using HapMap II dataset. We extended this model to involve 29 disjoint decision trees over 149 SNPs, and showed that this ensemble has an accuracy of $\geq 99.9 \%$, even if some of those 149 SNP values were missing. On an independent dataset, predominantly of Caucasian origin, our continental classifier showed $96.8 \%$ accuracy and improved genomic control's $\lambda$ from 1.22 to 1.11. We next used the HapMap III dataset to learn classifiers to distinguish European subpopulations (North-Western vs. Southern), East Asian subpopulations (Chinese vs. Japanese), African subpopulations (Eastern vs. Western), North American subpopulations (European vs. Chinese vs. African vs. Mexican vs. Indian), and Kenyan subpopulations (Luhya vs. Maasai). In these cases, ETHNOPRED produced ensembles of 3, 39, 21, 11, and 25 disjoint decision trees, respectively involving 31, 502, 526, 242 and 271 SNPs, with 10 -fold cross validation accuracy of $86.5 \% \pm 2.4 \%, 95.6 \% \pm 3.9 \%, 95.6 \% \pm 2.1 \%, 98.3 \% \pm 2.0 \%$, and $95.9 \% \pm 1.5 \%$. However, ETHNOPRED was unable to produce a classifier that can accurately distinguish Chinese in Beijing vs. Chinese in Denver.
\end{abstract}

Conclusions: ETHNOPRED is a novel technique for producing classifiers that can identify an individual's continental and sub-continental heritage, based on a small number of SNPs. We show that its learned classifiers are simple, cost-efficient, accurate, transparent, flexible, fast, applicable to large scale GWASs, and robust to missing values.

\footnotetext{
* Correspondence: sambasivarao.damaraju@albertahealthservices.ca

${ }^{\dagger}$ Equal contributors

${ }^{3}$ Department of Laboratory Medicine and Pathology, University of Alberta,

Edmonton, Alberta, Canada

${ }^{5}$ Cancer Care, Alberta Health Services, Edmonton, Alberta, Canada

Full list of author information is available at the end of the article
} 


\section{Background}

\section{Single nucleotide polymorphisms}

Single nucleotide polymorphisms (SNPs) as single base substitutions in DNA are the most common type of genetic variation in humans. SNPs are evolutionarily conserved and heritable. They give rise to one or more allelic variations at a loci and may confer phenotypic variance. Polymorphisms result from the evolutionary processes, and are modified by natural selection. They are common in nature and are related to biodiversity, genetic variation, and adaptation [1]. To date, millions of human SNPs have been identified and recorded in public databases such as dbSNP [2] or Ensembl [3].

\section{Genome wide association studies}

A genome wide association study (GWAS) is an examination of a large set of common genetic variants, such as SNPs, over a set of "labeled" individuals, seeking variants that are associated with a phenotype, such as disease susceptibility, disease prognosis or drug response under the "Common Disease-Common Variant" hypothesis [4,5]. A GWAS normally compares the DNA of two groups of participants: subjects who expressed a phenotype (cases) versus subjects who did not (controls). Here, the researcher compares the values of each individual feature (e.g., specific SNP) in the cases, with the corresponding values for this feature in the controls. If the range of values in these subgroups is significantly different, this feature is said to be associated with the phenotype. In contrast to candidate gene polymorphism studies which test only a few pre-defined genetic regions, GWASs investigate the entire genome [6,7]. The database of genotypes and phenotypes (dbGaP) [8] and the catalogue of published GWASs [9] archive and distribute the findings from GWASs to the broader scientific community.

\section{Population stratification}

Population stratification (aka population structure) is the presence of a systematic difference in allele frequencies between populations or subpopulations possibly due to different ancestry. We observe population stratification because of the differences in social history, ancestral patterns of geographical migration, mating practices, reproductive expansions and bottlenecks of different human subpopulations [10].

\section{Population stratification in GWASs}

While conducting a GWAS, a major concern is the possibility of inducing false positive or false negative associations between a SNP and the phenotype due to population stratification. This has motivated many researchers to consider techniques to address population stratification problem. As a pre-processing step in GWAS, these techniques either exclude some of the study subjects to alleviate the problem or adjust some of the SNPs to correct for population structure [11]. Here we review some of the standard techniques used to deal with population stratification problem in GWASs and discuss their limitations:

\section{Self-declared ancestry}

Many studies ask subjects to identify their own ethnicity, by reporting their ancestry and country of origin. Then they address the problem of population stratification by including the cases and controls that have the same self-reported ancestry and by excluding other subjects from the GWAS. However this method is sometimes misleading as some people might not know their full lineage information, or simply are mistaken. Furthermore, self-declared ancestry is not always sufficient to control population stratification as nearly all populations are confounded by genetic admixture at some level [12].

\section{Ancestry informative markers}

Some projects attempt to estimate ancestry using a panel of ancestry informative markers (AIMs) that show the highest absolute value difference in allele frequency between two ancestral populations. A small set (typically tens to hundreds) of well-established AIMs can perfectly distinguish continental differences between individuals [13-16]; however, panels of AIMs, described thus far, are less informative in detecting sub-continental differences in closely related populations such as Europeans [17-25].

\section{Genomic control}

A widely used approach to evaluate whether a dataset is confounded due to population stratification involves computing the genomic control $\lambda$, which is defined as the median $\chi^{2}$ (1 degree of freedom) association statistic across SNPs, divided by its theoretical median under the null distribution. A value of $\lambda \approx 1$ indicates no stratification, whereas $\lambda>1$ indicates population stratification or other confounders [26-29]. Despite its widespread application, genomic control method has a fundamental limitation. In the real world, some markers differ in their allele frequencies across ancestral populations more than others while the genomic control corrects for stratification by adjusting association statistics at each marker using a uniform overall inflation factor. This uniform adjustment is not sufficient to deal with both markers that have strong differentiation across ancestral populations and also those with smaller differentiation.

\section{Structured association}

Structured association techniques are unsupervised learning (clustering) methods such as STRUCTURE [30], which is based on a Bayesian framework, and latent class analysis [31], which is based on maximum-likelihood, that 
assign subjects of a case-control study cohort to discrete subpopulations based on their inter-cluster similarities and intra-cluster dissimilarities [32,33]. Although structured association methods have the advantage of assigning samples into meaningful population groups, they cannot be applied to GWAS datasets because of their intensive computational cost on large datasets provided by recent high-throughput measurements.

\section{Principal component analysis}

Techniques based on principal component analysis (PCA) [34-36], like EIGENSTRAT [34], are currently the state-of -the-art methods used in GWASs for population stratification correction. The EIGENSTRAT algorithm applies PCA to genotype data to infer continuous axes of genetic variations represented by principal component vectors and then adjusts genotypes and phenotype by amounts attributable to ancestry along each axis. Despite the widespread application of such PCA-based techniques, they have some disadvantages: First, they are not cost-efficient since they require genotyping thousands to millions of markers to be able to calculate principal component vectors. Second, to infer ancestry of subjects they apply PCA, a black-box model, which is not human readable (i.e., not transparent). Third, as high-throughput measurements produce many missing values, the straightforward PCA does not apply, leading EIGENSTRAT to use missing value imputation. However, such imputation techniques can be problematic in population genetics as they ignore interindividual and inter-ethnic variations, meaning such imputed datasets can lead to spurious association findings [37]. Fourth, the genotyping errors (GEs) that arise in highthroughput SNP measurements are a major issue in association studies [38-44] and substantially affect the efficiency of PCA-based methods like EIGENSTRAT [45].

\section{The purpose of our research study}

In this paper, we introduce a novel method, ETHNOPRED, for producing models that can accurately place subjects within continental and sub-continental populations, by applying a supervised learning (classification) technique to datasets from the second and third phases of the international HapMap project [46]. The resulting classifiers can help correct population stratification in association studies, overcoming some of the limitations of the conventional methods listed above. First, self-declared ancestry information is often problematic, except possibly for isolated populations with extensive inbreeding. ETHNOPRED does not rely on self-declared ancestry information and analyzes an individual's genome to properly identify his/her ancestry. Second, while small panels of AIMs for continental population identification are designed, panels of AIMs for subcontinental population identification, if designable, either are less informative or use a large set of markers. However,
ETHNOPRED produces accurate classifiers not only for continental population detection but also for subcontinental population detection using a small number of markers. Third, ETHNOPRED does not rely on the assumption made by the genomic control method that all markers contribute equally to population stratification and instead benefits from the fact that different markers ontribute to population differences in different degrees. Fourth, unlike structured association methods, ETHNOPRED classifiers are fast and easily applicable to the large GWAS datasets generated by high-throughput measurement techniques like microarrays and next generation sequencers. Fifth, ETHNOPRED classifiers require genotyping of only tens to hundreds of SNPs for accurate population identification. Hence they are simpler and more cost-efficient than PCA-based methods, which require genotyping of thousands to millions of SNPs. Sixth, PCA based methods like EIGENSTRAT are substantially affected by the genotyping errors that arise in highthroughput SNPs measurements. However, low-throughput SNP measurements of tens to hundreds of SNPs required by ETHNOPRED classifiers may be easily validated on independent genotyping platforms to rule out genotyping errors and assess concordance of genotype calls across independent platforms. Once these criteria are established, these selected SNP panels could be used to identify population stratification across projects sharing similar cases and control cohorts in molecular epidemiological studies. Seventh, ETHNOPRED classifiers are a set of easy-to-read rules. Thus unlike PCA-based methods, these classifiers are transparent, and so can provide insight into the population classification problem they are dealing with. Eighth, unlike PCA-based methods, ETHNOPRED classifiers do not require any kind of imputation to handle missing values. ETHNOPRED classifiers are robust to missing values as their ensemble structure allows them the flexibility to deal with missing SNPs by simply removing some decision trees, and still remain able to accurately identify ancestry.

\section{Methods}

\section{Datasets}

Our objective is to build predictive tools to determine an individual's continental and sub-continental ancestry based on the values of a small set of his/her SNPs. We develop this tool by applying supervised learners to datasets from the second and third phases of the international HapMap project. The HapMap project is a multi-country effort to identify and catalogue genetic similarities and differences in human beings and to determine the common patterns of DNA sequence variations in the human genome. It is developing a map of these patterns across the genome by determining the genotypes of more than a million sequence variants, 


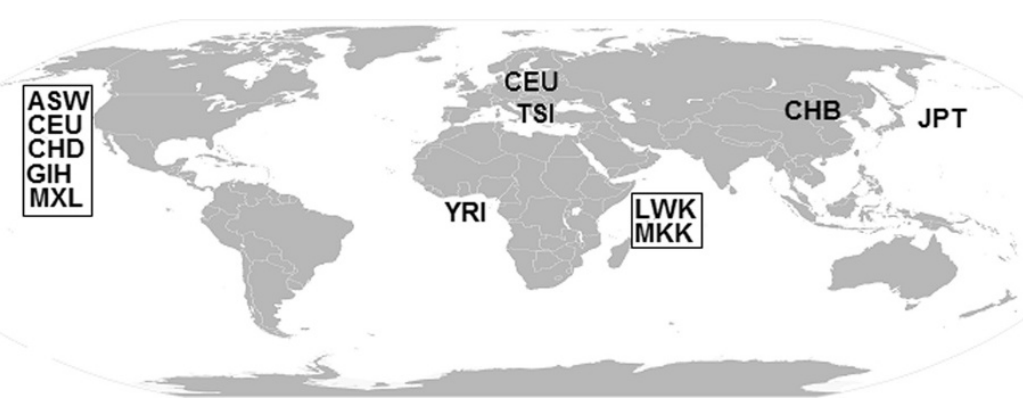

Figure 1 Geographic map of the HapMap phase III world populations. ASW = Southwest USA residents with African ancestry; CEU = Utah residents with Northern and Western European ancestry; CHB = Han Chinese in Beijing, China; CHD = Chinese in Metropolitan Denver, Colorado; $\mathrm{GIH}=$ Gujarati Indians in Houston, Texas; JPT = Japanese in Tokyo, Japan; LWK = Luhya in Webuye, Kenya; MKK = Maasai in Kinyawa, Kenya; MXL = Mexicans in Los Angeles, California; TSI = Toscani in Italia; YRI = Yoruba in Ibadan, Nigeria.

their frequencies and the degree of association between them, in DNA samples from subpopulations with ancestry from East and West Africa, East Asia, North and West Europe, and North America.

The HapMap phase II datasets, released in 2007, contained 270 subjects - including 90 Utah residents with ancestry from Northern and Western Europe (CEU), 90 Yorubans from Ibadan, Nigeria (YRI), and a mixture of 45 Japanese in Tokyo and 45 Han Chinese in Beijing (JPT/ CHB) - each genotyped on an Affymetrix SNP array 6.0 platform, measuring 906600 SNPs. We utilize the HapMap II datasets to build a predictive model for inferring the continental ancestry origins (West Africa vs. East Asia vs. North-West Europe) of an individual. We apply the resulting classifier to a dataset of 696 breast cancer study subjects (348 breast cancer cases and 348 apparently healthy controls) from Alberta, Canada, genotyped on the same Affymetrix SNP array platform. We have selfdeclared ancestry of these 348 control individuals. These study subjects provided written informed consent and the study was approved by the Alberta Cancer Research Ethics Committee of the Alberta Health Services [47].

The HapMap phase III datasets, released in 2009, contained 1458387 SNPs of 1397 subjects including 87 Southwest USA residents with African ancestry (ASW), 165 Utah residents with ancestry from Northern and

\section{Table 1 Pre-processing statistics of continental population classification problem based on HapMap Phase II samples}

\begin{tabular}{lr}
\hline SNP groups & Number of SNPs \\
\hline All SNPs & 906600 \\
SNP with Call Rate $<100 \%$ & 186578 \\
SNPs on Non-autosomal Chromosomes & 38306 \\
SNPs Deviated from HWE & 184854 \\
Filtered SNPs & 295454 \\
Unfiltered SNPS & 611146 \\
\hline
\end{tabular}

Western Europe (CEU), 137 Han Chinese in Beijing, China (CHB), 109 metropolitan Denver, Colorado residents with Chinese ancestry (CHD), 101 Gujarati Indians in Houston, Texas (GIH), 113 Japanese in Tokyo, Japan (JPT), 110 individuals from Luhya tribe in Webuye, Kenya (LWK), 86 Los Angeles, California residents with Mexican ancestry (MXL), 184 individuals from Maasai tribe in Kinyawa, Kenya (MKK), 102 Toscani Italians (TSI), and 203 Yorubans in Ibadan, Nigeria (YRI). Figure 1 shows the geographic map of the HapMap III world populations. We utilize the HapMap III datasets to build predictive models for infering sub-continental ancestry origins of Africans (LWK vs. MKK vs. YRI), Europeans (CEU vs. TSI), East Asians (CHB vs. JPT), North Americans (ASW vs. CEU vs. CHD vs. GIH vs. MXL), Kenyans (LWK vs. MKK), and Chinese (CHB vs. CHD).

\section{Pre-processing}

The allele with the dominant occurrence within a population is called the major allele (A), while the allele occurring less frequently is called the minor allele (B). Together, the alleles from paternal and maternal chromosomal loci can produce three distinct genotypes: When both alleles (ie, inherited from both parents) are the major alleles (A_A), the genotype is called wild type homozygous; when both the inherited alleles are minor (B_B), the genotype is called variant type homozygous; and when the two alleles are different (A_B), the genotype is called heterozygous.

To build our continental population classifier, we first identified the relevant SNPs from the HapMap II dataset, by removing a SNP if (a) it has a NoCall for any of the 270 subjects; (b) it is located on the X, Y, mitochondria (MT), or on an unknown chromosome; or (c) its genotype frequency deviates significantly from Hardy-Weinberg equilibrium (HWE) proportions, tested with Pearson's chi-squared $\left(x^{2}\right)$ test (nominal p-value $<0.05$ ) [48]. We used criteria (a) to train our model using SNPs without missing values; (b) so the tool would be applicable to anyone, regardless of gender; and (c) by reasoning that 
observed genotype frequencies that deviate from HWE do not match the expected distributions of alleles, and hence are not reliable. These pre-processing steps removed a total of 295454 SNPs, leaving 611146 SNPs amenable for further scrutiny. Table 1 summarizes the statistics of the SNPs removed in the pre-processing steps, applied on HapMap II datasets.

To build our sub-continental population classifiers, we followed similar filtering criteria on HapMap III dataset. These pre-processing steps respectively removed 841790, 565554, 575492, 931993, 677326, and 629023 SNPs, and left 616597, 892833, 882895, 526394, 781061, and 829364 SNPs amenable for further analysis for the African, East Asian, European, North American, Kenyan, and Chinese population classification problems. Table 2 summarizes the statistics of the SNPs removed in the pre-processing steps, applied on HapMap III datasets.

\section{Predictive modelling}

Machine learning provides a variety of statistical, probabilistic, and optimization techniques to analyze and interpret data, which allow computers to autonomously learn from past examples by finding patterns to form predictive models - often finding hard-to-discern patterns, from noisy and complex datasets [49-51]. Machine learning has been applied successfully in many areas: Baldi and Brunak [52], Larranga et al. [53], and Tarca et al. [54] each surveyed various applications of machine learning in biology, medicine, and genetics including gene finding [55], eukaryote promoter recognition [56], protein structure prediction [57], pattern recognition in microarrays [58], gene regulatory response prediction [59], and protein/gene identification in text [60]. Herein, we learn a sequence of CART decision trees for continental and sub-continental population identification [61,62]. While machine learning provides many systems for learning classifiers, we focus on decision trees as these learners are easy to use (as they do not require the user to provide any input parameters) and relatively fast to train, and the resulting classifiers run quickly and are easy to interpret (which may explain why they are widely applied in biological/medical domains).

"Ensemble learning" refers to a class of machine learning methods that combine the individual decisions of a set of learned "base predictors" to obtain a better predictive performance [63]. In general, an ensemble of predictors will be more accurate than any of its individual members if the constituent predictors are individually accurate and collectively diverse [64]. Ensemble models have been successfully applied on high-dimensional datasets generated by novel "omics" measurements, such as gene expression microarrays [65,66]. Many ensemble techniques such as bagging, boosting, AdaBoost, and stacking - rely on manipulation of the input dataset by sampling of subjects or sampling of features, then learning individual base classifiers on these subsets of the input dataset [67]. While the main goal of ensemble predictors is to produce an accurate classifier (as the ensemble can sometimes

Table 2 Pre-processing statistics of HapMap phase III datasets and sub-continental population classification problems

\begin{tabular}{|c|c|c|c|c|c|c|c|}
\hline Dataset/Problem & Samples & All SNPs & $\begin{array}{l}\text { SNPs with call } \\
\text { rate }<100 \%\end{array}$ & $\begin{array}{l}\text { SNPs on Non- } \\
\text { autosomal Chr. }\end{array}$ & $\begin{array}{l}\text { SNPs deviated } \\
\text { from HWE }\end{array}$ & Filtered SNPs & $\begin{array}{l}\text { Unfiltered } \\
\text { SNPs }\end{array}$ \\
\hline ASW & 87 & 1458387 & 214898 & 34554 & 94234 & 298524 & 1159863 \\
\hline CEU & 165 & 1458387 & 376531 & 34554 & 81633 & 427638 & 1030749 \\
\hline $\mathrm{CHB}$ & 137 & 1458387 & 353208 & 34554 & 77028 & 423270 & 1035117 \\
\hline CHD & 109 & 1458387 & 352031 & 34554 & 77111 & 421328 & 1037059 \\
\hline GIH & 101 & 1458387 & 234863 & 34554 & 85463 & 314376 & 1144011 \\
\hline JPT & 113 & 1458387 & 271105 & 34554 & 75502 & 337033 & 1121354 \\
\hline LWK & 110 & 1458387 & 365638 & 34554 & 97174 & 425375 & 1033012 \\
\hline MKK & 184 & 1458387 & 411395 & 34554 & 105490 & 471384 & 987003 \\
\hline MXL & 86 & 1458387 & 311704 & 34554 & 86910 & 387207 & 1071180 \\
\hline TSI & 102 & 1458387 & 268916 & 34554 & 81919 & 326585 & 1131802 \\
\hline YRI & 203 & 1458387 & 423100 & 34554 & 94449 & 476513 & 981874 \\
\hline European & 267 & 1458387 & 493449 & 34554 & 137488 & 575492 & 882895 \\
\hline East Asian & 250 & 1458387 & 475217 & 34554 & 129695 & 565554 & 892833 \\
\hline African & 497 & 1458387 & 742671 & 34554 & 228268 & 841790 & 616597 \\
\hline North American & 548 & 1458387 & 803678 & 34554 & 306572 & 931993 & 526394 \\
\hline Kenyan & 294 & 1458387 & 590202 & 34554 & 170547 & 677326 & 781061 \\
\hline Chinese & 246 & 1458387 & 538224 & 34554 & 131394 & 629023 & 829364 \\
\hline
\end{tabular}


overcome the over-fitting problem reported for decision trees in high-dimensional problems [68]), we used this approach to produce a classifier that is robust to missing SNP values. Our system therefore learns a set of disjoint trees; we later explain how this allows the classifier to predict the label of a subject, even if that subject is missing many SNP values.

Here we explain how ETHNOPRED learns an ensemble of disjoint decision trees, focusing on continental population classifier case. It first applies the CART learning algorithm to the dataset of 270 subjects over the 611146 SNPs mentioned above, to produce the decision tree (Figure 2) with 3 internal nodes (each a condition on a specific SNP) and 4 leaf nodes (class labels), corresponding to the 4 rules shown in Figure 2. It then removes these 3 SNPs from the list of 611146 SNPs and applies the same CART decision tree learning algorithm to the dataset of 270 subjects and the remaining 611143 SNPs, to produce a second decision tree. We repeat this algorithm, each time removing the SNPs used in the previous trees, to produce the next decision tree.

The ETHNOPRED continental population classifier learns $\mathrm{N}=29$ disjoint decision trees. We explain below that $\mathrm{N}=29$ guarantees that this system is robust against missing SNP values - that is, based on some simple assumptions, we anticipate that at least $99.9 \%$ of the subjects will include calls on the SNPs needed to "match" several decision trees; enough trees that the

\section{Continental Classification Based on Hapmap2 Dataset} (CEU vs. YRI vS. CHB/JPT)

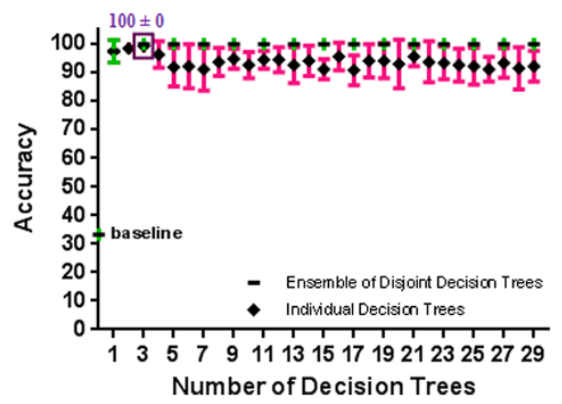

Figure 3 A comparison of 10 -fold cross validation accuracy of individual decision trees and ensembles of disjoint decision trees of variable size in continental population classification problem using HapMap phase II datasets. An ensemble of 3 disjoint decision trees involving 10 SNPs has a 10-fold cross validation accuracy of $100 \%$ which is significantly better than the baseline accuracy of $33.3 \%$.

resulting sub-ensemble will be at least $99.9 \%$ accurate. This analysis appears below.

Additional file 1: Appendix A and Figure 3 show the estimated accuracies of the first $\mathrm{k}$ decision tree: the first tree, alone, is $97.41 \%$ and the ensemble classifier using the first 3 decision trees is $100 \%$. If accuracy was our only concern, our ensemble classifier would just use these 3 decision trees, involving its 10 SNPs. However,

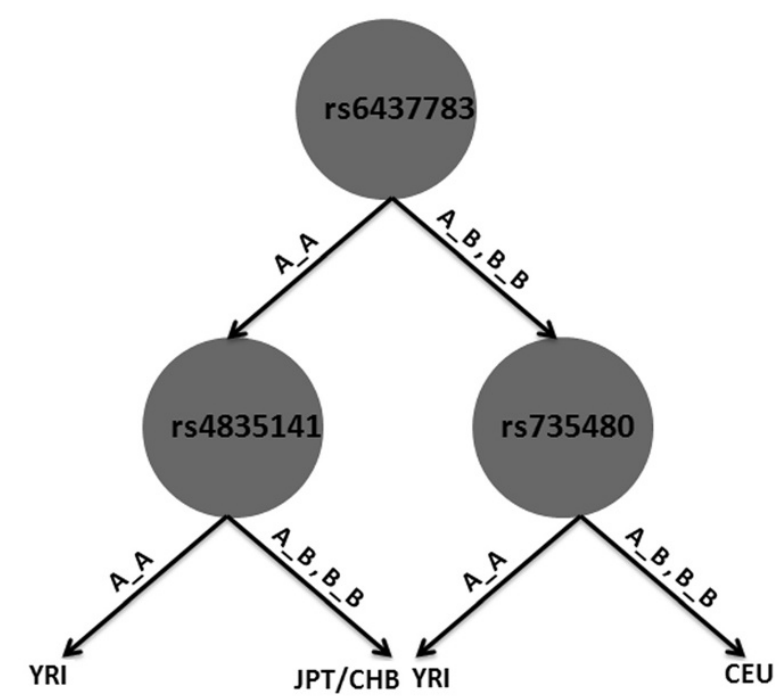

Rule 1: IF rs6437783€\{'A_A' $\}$ AND rs 4835141 $€\{$ 'A_A' $\}$ THEN ethnicity is ' $Y R{ }^{\prime}$

Rule 2: IF rs6437783€\{'A-A'\} AND rs $4835141 \epsilon\{$ 'A B', 'B B' $\}$ THEN ethnicity is 'JPT/CHB'

Rule 3: IF rs6437783 $\{\{$ 'A_B', 'B_B' $\}$ AND rs $735480 €\{$ 'A_A' $\}$ THEN ethnicity is 'YRI'

Rule 4: IF rs6437783 $€\{$ 'A_B','B_B' $\}$ AND rs735480 $€\{$ 'A_B', 'B_B' $\}$ THEN ethnicity is 'CEU'

Figure 2 The first decision tree and associated rule-set of the continental classifier produced by ETHNOPRED algorithm. The decision tree uses 3 internal nodes (SNPs) acting as decision criterions and 4 external nodes (populations) demonstrating decisions. The number of rules in the relevant rule-base is equal to the number of external nodes of the decision tree. 
this 3 decision tree system can only classify a subject if that subject includes values for (essentially) all 10 SNPs. Missing genotype data is a common problem in genotyping experiments, due to assay design failures, platform specific differences in the SNPs analyzed or due to hybridization artifacts in these high-throughput array platforms [69]. Here, we show that $\mathrm{N}=29$ decision trees are sufficient, under mild assumptions, to obtain an accuracy (Acc) of $\geq 99.9 \%$ with $99.9 \%$ confidence (C), even considering missing SNPs: We trained 30 disjoint decision trees and found the average number of SNPs used in these 30 decision trees is $n=154 / 30 \approx 5.13$. We

\section{Table 3 The average 10-fold cross validation accuracy of ensembles of size $m$, for $m=1 . .30$ in continental population classification problem}

\begin{tabular}{|c|c|c|}
\hline $\begin{array}{l}\text { Number of models } \\
\text { in the ensemble }(\mathrm{m})\end{array}$ & $\begin{array}{l}\text { Number of } \\
\text { ensembles }\end{array}$ & $\begin{array}{l}\text { The average } 10 \text {-fold cross } \\
\text { validation accuracy of } \\
\text { ensembles of size } m \text { (Acc) }\end{array}$ \\
\hline 1 & 30 & 95.38 \\
\hline 2 & 435 & 91.34 \\
\hline 3 & 4060 & 98.36 \\
\hline 4 & 27405 & 97.03 \\
\hline 5 & 142506 & 99.32 \\
\hline 6 & 593775 & 98.81 \\
\hline 7 & 2035800 & 99.67 \\
\hline 8 & 5852926 & 99.44 \\
\hline 9 & 14300000 & 99.93 \\
\hline 10 & 30000000 & 99.92 \\
\hline 11 & 54600000 & 99.98 \\
\hline 12 & 86500000 & 99.96 \\
\hline 13 & 120000000 & 99.99 \\
\hline 14 & 145000000 & 99.99 \\
\hline 15 & 155000000 & 99.99 \\
\hline 16 & 145000000 & 99.99 \\
\hline 17 & 120000000 & 99.99 \\
\hline 18 & 86500000 & 99.99 \\
\hline 19 & 54600000 & 99.99 \\
\hline 20 & 30000000 & 99.99 \\
\hline 21 & 14300000 & 99.99 \\
\hline 22 & 5852926 & 99.99 \\
\hline 23 & 2035800 & 99.99 \\
\hline 24 & 593775 & 99.99 \\
\hline 25 & 142506 & 99.99 \\
\hline 26 & 27405 & 99.99 \\
\hline 27 & 4060 & 99.99 \\
\hline 28 & 435 & 99.99 \\
\hline 29 & 30 & 99.99 \\
\hline 30 & 1 & 100 \\
\hline
\end{tabular}

then assumed that, for the Affymetrix genome wide SNP array 6.0 platform, NoCall's are independent from one SNP to another, and that the probability that a SNP value will be a NoCall is at worst $\mathrm{u}=0.1$ (based on assessment on the HapMap II dataset). This means that the probability that a subject will include all of the SNPs for a decision tree is $\mathrm{p} \leq(1-\mathrm{u})^{\mathrm{n}}=0.9^{5.13}=0.59049$, and so the probability that a subject will not include all of the SNPs of a decision tree is at least $\mathrm{q}=1-\mathrm{p}=0.40951$. We now ask how many decision trees $(\mathrm{m})$ are needed to insure that the average accuracy (Acc) of any subset of $m$ trees is at least $99.9 \%$. We therefore considered a sampling of ensembles of size 1 (i.e., individual decision trees) and

Table 4 The confidence of having $m=9$ decision trees without missing SNPs for $N=1 . .30$ in continental population classification problem

\begin{tabular}{|c|c|}
\hline Number of decision trees $(\mathrm{N})$ & $\begin{array}{l}\text { Confidence of having } m=9 \text { decision } \\
\text { trees with no missing SNPs (C) }\end{array}$ \\
\hline 1 & 0 \\
\hline 2 & 0 \\
\hline 3 & 0 \\
\hline 4 & 0 \\
\hline 5 & 0 \\
\hline 6 & 0 \\
\hline 7 & 0 \\
\hline 8 & 0 \\
\hline 9 & 0.873 \\
\hline 10 & 4.09 \\
\hline 11 & 10.676 \\
\hline 12 & 20.566 \\
\hline 13 & 32.716 \\
\hline 14 & 45.652 \\
\hline 15 & 58.013 \\
\hline 16 & 68.86 \\
\hline 17 & 77.744 \\
\hline 18 & 84.616 \\
\hline 19 & 89.682 \\
\hline 20 & 93.265 \\
\hline 21 & 95.71 \\
\hline 22 & 97.328 \\
\hline 23 & 98.369 \\
\hline 24 & 99.023 \\
\hline 25 & 99.424 \\
\hline 26 & 99.666 \\
\hline 27 & 99.809 \\
\hline 28 & 99.892 \\
\hline 29 & 99.94 \\
\hline 30 & 99.967 \\
\hline
\end{tabular}


calculated the average 10 -fold cross validation accuracy. We next computed the average 10 -fold cross validation accuracy over a sample of pairs of decision trees; then over triples, and so forth, for $\mathrm{i}=1 . .30$ (Table 3). We found that $\mathrm{m}=9$ is sufficient to obtain an average 10-fold cross validation accuracy (Acc) of 99.9\%.

The next challenge was in determining how many trees $(\mathrm{N})$ are necessary, to be confident that the SNPs for $99.9 \%$ of all subjects will include calls on all of the SNPs for at least 9 trees.The probability of having at least $m$ decision trees with no missing SNPs, given $\mathrm{N}$ decision trees, with probability $\mathrm{p}$ that a decision tree includes only specified SNPs, is:

$$
C=\left(1-\sum_{i=1}^{8}\left(\begin{array}{c}
N \\
i
\end{array}\right) * p^{i} *(1-p)^{N-i}\right)
$$

Table 4 shows the values for $\mathrm{C}$ based on different values for $\mathrm{N}$; here, we see $\mathrm{N}=29$ decision trees is sufficient to have $99.9 \%$ confidence (C) that a subject will include all of the SNPs in at least $m=9$ decision trees, which our earlier experiments show is sufficient to produce an accuracy of $\geq 99.9 \%$. Additional file 2 : Appendix B summarizes this analysis.

\section{Models' usage for population stratification correction}

For each continental and sub-continental ancestry identification problem, the pre-processing and predictive modeling steps produce a model (i.e., in the case of continental classification problem, the model is an ensemble of 29 decision trees) that can be used to classify novel subjects. For example, in continental population identification, we need to only find the values $\{$ A_A, A_B, B_B, NoCall $\}$ of the relevant 149 SNPs, then hand this set of 149 values to each of the 29 decision trees. Each tree involves a small number of SNPs (typically 3-7); if they are all specified (that is, none are "NoCall") for a novel subject, this tree will produce a predicted label - one of the three ethnicity groups: CEU, YRI, or CHB/JPT. If not, the tree makes no prediction. This will lead to a set of at-most-29 predicted ethnicity values for this subject. As no human population is homogenous, given a novel subject with unknown

Table 5 Comparison of self-declared lineage information and ETHNOPRED's result on 348 controls selected for a breast cancer susceptibility study of Caucasian women of Alberta, Canada [45]

\begin{tabular}{lll}
\hline & $\begin{array}{l}\text { ETHNOPRED } \\
\text { predicts as CEU }\end{array}$ & $\begin{array}{l}\text { ETHNOPRED predicts } \\
\text { as non-CEU }\end{array}$ \\
\hline $\begin{array}{l}\text { Self-declared lineage } \\
\text { information as CEU }\end{array}$ & 330 & 0 \\
$\begin{array}{l}\text { Self-declared lineage } \\
\text { information as non-CEU }\end{array}$ & 11 & 7 \\
\hline
\end{tabular}

ancestry, our model can provide a vector of population inclusion probabilities.

For example, when classifying a novel person with the initial continental classification, imagine 15 trees vote for CEU, 4 for YRI, 8 for JPT/CHB, and 2 are silent; this would produce the vector $(15 / 27,4 / 27,8 / 27)$. These vector-valued predictions provide flexibility for researchers conducting a GWAS, as they can then, for example, define cut-off criterion for including a subject within a population under study. For each subject, continental classifier then returns, as ethnicity label, the ethnicity with the largest number of trees. In the Results section, we explain such panels for resolving the population stratification problem in closely related populations within a continent or a country as well.

\section{Evaluation}

We built the ETHNOPRED classifiers using HapMap II and HapMap III datasets as training data. Before using each classifier, we estimated its quality using a 10-fold cross validation $(\mathrm{CV})$ [70]. This meant partitioning the training dataset into 10 disjoint folds. Each time we used nine of these folds $\left(9 / 10^{\text {th }}\right.$ of data) as training set for learning a sequence of decision trees, applying the algorithm explained in the Predictive Modeling section. We then used the remaining fold $\left(1 / 10^{\text {th }}\right.$ of data) as a test set; here to compute, for each subject, class labels (one from each decision tree), and also the majority vote over these model (corresponding to the ensemble classifier). As we knew the true label for these subjects, we then obtained an accuracy score (the percentage of correct predictions over the total number of predictions) for each of the disjoint decision trees and for the final ensemble. We repeated this process 10 times, each time measuring accuracy of the predictors on a different fold. We estimated the final accuracy of the decision trees and ensemble model as an average of these 10 folds, with variance based on the spread of these 10 numbers. We used a similar way to evaluate the quality of the ETHNOPRED(k) classifier, where each such classifier was involved in returning the majority vote over subsequence of $\mathrm{k}$ individual decision trees.

Table 6 Comparison of self-declared lineage information and EIGENSTRAT's result on $\mathbf{3 4 8}$ controls selected for a breast cancer susceptibility study of Caucasian women of Alberta, Canada [45]

\begin{tabular}{lll}
\hline & $\begin{array}{l}\text { EIGENSTRAT } \\
\text { predicts as CEU }\end{array}$ & $\begin{array}{l}\text { EIGENSTRAT predicts } \\
\text { as non-CEU }\end{array}$ \\
\hline $\begin{array}{l}\text { Self-declared lineage } \\
\text { information as CEU }\end{array}$ & 321 & 0 \\
$\begin{array}{l}\text { Self-declared lineage } \\
\text { information as non-CEU }\end{array}$ & 9 & 18 \\
\hline
\end{tabular}




\section{Results and discussion}

\section{Continental ancestry identification}

Table 1 summarizes the statistics of the SNPs removed in the pre-processing step, which recall filtered out each SNP with a call rate of less than $100 \%$, or that are located on X, Y, MT, or an unknown chromosome, or deviated from the HWE; this removed 295454 SNPs, leaving 611146 SNPs for further analyses.

The final ensemble model, learned from all 270 subjects of the HapMap Phase II datasets, was composed of 29 disjoint decision trees, which each involved between 3 to 7 SNPs and between 4 to 8 leaf nodes/rules. This corresponds to a total of 178 rules involving 149 SNPs in the ensemble model (see Additional file 3: Appendices C, Additional file 4: Appendix D and Additional file 5: Appendix E). Additional file 1: Appendix A and Figure 3 present the 10-fold cross validation (CV) accuracy of the disjoint decision trees built based on the ETHNOPRED algorithm showing the mean of the 10-fold CV accuracy of these models was between $90.7 \%$ and $99.3 \%$. We see that the ensemble over only the first tree had a mean accuracy of $97.4 \%$; the accuracy decreased (albeit insignificantly) to $95.9 \%$ by adding the second tree; the ensemble over 3 (or more) trees was $100 \%$ accurate. While adding additional trees to the ensemble did not improve the accuracy, our approach did increase its robustness to missing SNP values, as it means ETHNOPRED can produce a classification label even if the subject did not have calls on all 149 SNPs. Recall that ETHNOPRED can classify most subjects with missing SNP values as it can ignore any tree that includes missing SNPs, and returns as label the majority vote of the remaining trees.

To further assess the accuracy of ETHNOPRED, we also used a hold-out set of 696 breast cancer subjects (348 breast cancer cases and 348 controls) genotyped in Alberta, Canada. We had self-declared ethnicity labels for the control subjects. Here, we compared our ETHNOPRED against the commonly-used EIGENSTRAT system, in terms

Table 7 Summary of the sub-continental classification problems results

\begin{tabular}{|c|c|c|c|c|c|c|}
\hline $\begin{array}{l}\text { Sub-continental } \\
\text { problem }\end{array}$ & $\begin{array}{l}\text { Number of } \\
\text { subjects, split }\end{array}$ & $\begin{array}{l}\text { Number } \\
\text { of SNPs }\end{array}$ & Baseline & $\begin{array}{l}\text { DT1 (Number of } \\
\text { SNPs), Accuracy }\end{array}$ & $\begin{array}{l}\text { Minimal Number of DTs } \\
\text { (Number of SNPs), Accuracy }\end{array}$ & $\begin{array}{l}\text { Number of Robust DTs } \\
\text { (Number of SNPs) }\end{array}$ \\
\hline \multirow[t]{3}{*}{ European } & 267 & 882895 & $61.8 \%$ & $1(10), 79.0 \% \pm 5.6 \%$ & $3(31), 86.6 \% \pm 2.4 \%$ & $15(180)$ \\
\hline & CEU: 165 & & & & & \\
\hline & TSI: 102 & & & & & \\
\hline \multirow[t]{3}{*}{ East Asian } & 250 & 892833 & $54.8 \%$ & $1(12), 74.4 \% \pm 7.9 \%$ & $39(502), 95.6 \% \pm 3.9 \%$ & $67(877)$ \\
\hline & CHB: 137 & & & & & \\
\hline & JPT: 113 & & & & & \\
\hline \multirow[t]{4}{*}{ African } & 497, & 616597 & $40.8 \%$ & $1(23), 66.2 \% \pm 5.3 \%$ & $21(526), 95.6 \% \pm 2.1 \%$ & 157 (4236) \\
\hline & LWK:110 & & & & & \\
\hline & MKK: 184 & & & & & \\
\hline & YRI: 203 & & & & & \\
\hline \multirow[t]{6}{*}{ North American } & 548 & 526394 & $30.1 \%$ & $1(19), 82.7 \% \pm 5.4 \%$ & 11 (242), 98.4\% $\pm 2.0 \%$ & $70(1643)$ \\
\hline & ASW: 87 & & & & & \\
\hline & CEU: 165 & & & & & \\
\hline & CHD: 109 & & & & & \\
\hline & GlH: 101 & & & & & \\
\hline & MXL: 86 & & & & & \\
\hline \multirow[t]{3}{*}{ Kenyan } & 294 & 781061 & $62.6 \%$ & $1(11), 79.2 \% \pm 3.5 \%$ & 25 (271), $95.9 \% \pm 1.5 \%$ & $31(341)$ \\
\hline & LWK: 110 & & & & & \\
\hline & MKK: 184 & & & & & \\
\hline \multirow[t]{3}{*}{ Chinese } & 246 & 829364 & $55.7 \%$ & $1(15), 47.2 \% \pm 9.1 \%$ & $-(-), \leq 55.7 \%$ & $-(-)$ \\
\hline & CHB: 137 & & & & & \\
\hline & CHD: 109 & & & & & \\
\hline
\end{tabular}

This table summarizes the result of our studies on various sub-continental classification problems. The "Number of Subjects, Split" column shows the total number of subjects, followed by the list of (ethnic-group; number) pairs, giving the name of each subgroups and its size here. The "Number of SNPs" column gives the number of SNPs used for this study. The "Baseline" column gives the baseline accuracy of just using the majority class. The "DT1 (Number of SNPs), Accuracy" column provides the number of SNPs in the first decision tree, and its estimated 10-fold cross-validation accuracy. The "Minimal Number of DTs (Number of SNPs), Accuracy" column gives the minimal number of disjoint decision trees required to achieve the highest accuracy, and the number of SNPs involved, in these trees. The "Number of Robust DTs (Number of SNPs)" column gives the number of decision trees required to achieve robustness and the number of SNPs involved. 


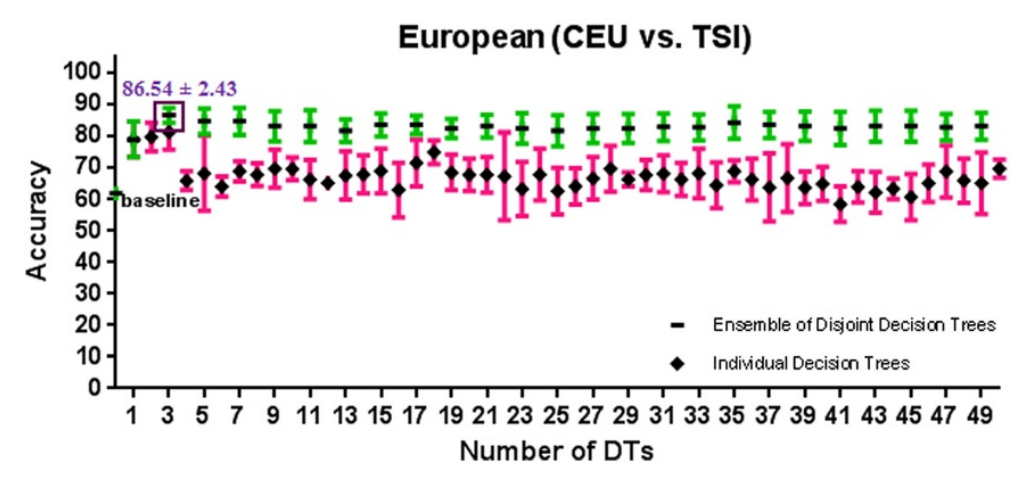

Figure 4 A comparison of 10-fold cross validation accuracy of individual decision trees and ensembles of disjoint decision trees of variable size in European population classification problem using HapMap phase III datasets. An ensemble of 3 disjoint decision trees involving 31 SNPs has a 10-fold cross validation accuracy of $86.5 \% \pm 2.4 \%$ which is significantly better than the baseline accuracy of $61.8 \%$.

of the prediction accuracy and genomic control inflation factor $(\lambda)$ improvement. Here, we extracted the values of ETHNOPRED's 149 SNPs for each subject. Note that 17 of these 149 SNPs had NoCalls for at least one subject. For each subject, each of ETHNOPRED's 29 decision trees predicted the subject's ethnicity to be one of "CEU", "YRI", "JPT/CHB", or "Missing". Continental classifier then calculates the covariate probability vector and returns the ethnicity with the majority vote as the predicted label for that subject. Additional file 6: Appendix F summarizes ETHNOPRED output for test dataset of 696 subjects. Prior knowledge of the subjects' ethnicity labels, when available, would help assess the predictive accuracies of ETHNOPRED (or EIGENSTRAT) - eg, many previously published studies (including our [45]) have used the HapMap subjects' self-declared ethnicity label to evaluate their ethnicity classifiers. We extrapolated this logic to calculate the prediction accuracies of ETHNOPRED over 348 control subjects, based on their self-declared ethnicity. Additional file 7: Appendix G summarizes the subjects' ethnicity labels, classified by ETHNOPRED (and the number of decision trees involved), EIGENSTRAT, and selfdeclared ethnicity label. Table 5 shows that ETHNOPRED's ethnicity classification matched closely with the subject's self-reported ethnicity (96.8\%); Table 6 provides similar statistics for EIGENSTRAT (97.4\%). The ETHNOPRED classifier labels 677 subjects as "CEU"; we could therefore use only these subjects and exclude the other 19 subjects for which either "YRI" or "CHB/JPT" is the majority ancestry covariate. Then we computed the inflation factor using the Genomic Control method for these subjects. For the entire sample size of 696 unclassified subjects in the association study, the computed inflation factor was 1.22, whereas the inflation factor computed for the 677 subjects classified as "CEU" by ETHNOPRED was 1.11, and the inflation factor for the 623 subjects classified as "CEU" by EIGENSTRAT was 1.10. While ETHNOPRED's learned classifier gives roughly the same improvement to the inflation factor as EIGENSTRAT, it offered the advantage of using a set of only 149 SNPs to achieve the classification of

\section{East Asian (CHB vs. JPT)}

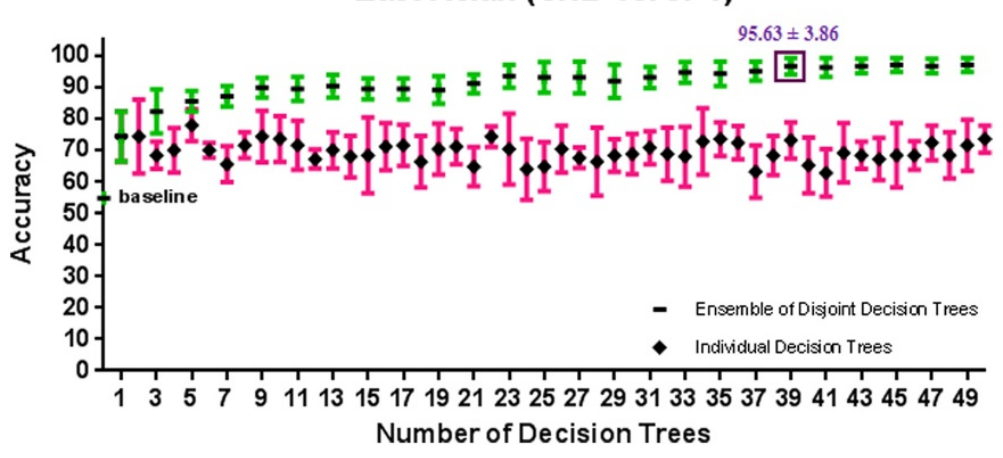

Figure 5 A comparison of 10-fold cross validation accuracy of individual decision trees and ensembles of disjoint decision trees of variable size in East Asian population classification problem using HapMap phase III datasets. An ensemble of 39 disjoint decision trees involving 502 SNPs has a 10-fold cross validation accuracy of $95.6 \% \pm 3.9 \%$ which is significantly better than the baseline accuracy of $54.8 \%$. 


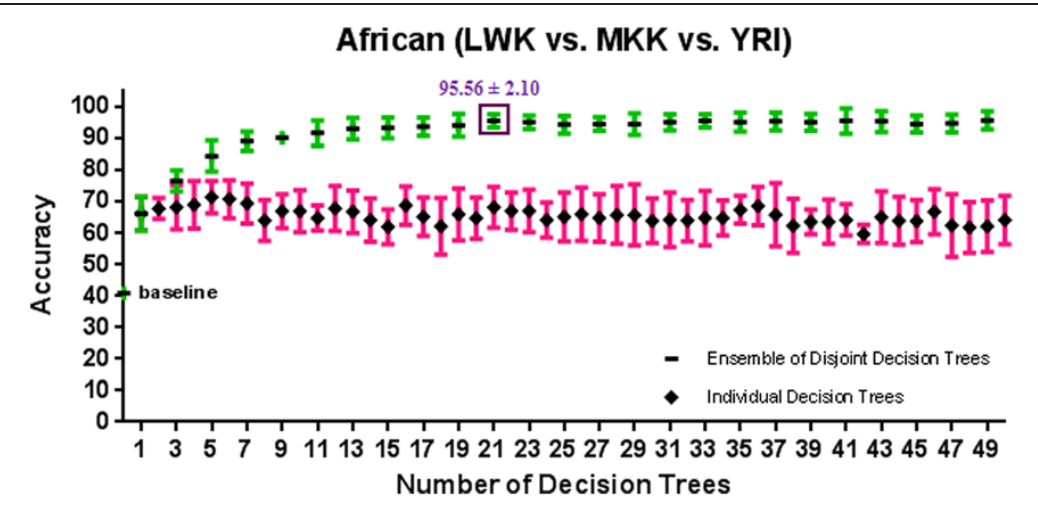

Figure 6 A comparison of 10-fold cross validation accuracy of individual decision trees and ensembles of disjoint decision trees of variable size in African population classification problem using HapMap phase III datasets. An ensemble of 21 disjoint decision trees involving 526 SNPs has a 10 -fold cross validation accuracy of $95.6 \% \pm 2.1 \%$ which is significantly better than the baseline accuracy of $40.8 \%$.

ethnicity label (CEU), which is significantly smaller than the 906,600 SNPs used by EIGENSTRAT.

\section{Sub-continental ancestry identification}

Table 2 summarizes the statistics of the SNPs filtered in the pre-processing step: those SNPs with a call rate of less than $100 \%$, or located on X, Y, MT, or on an unknown chromosome, or deviated from the HWE; starting with 1458387 SNPs in the HapMap III dataset, this filtering removed 493449, 475217, 742671, 803678, 590202, and 538224 SNPs respectively in European, East Asian, African, North American, Kenyan, and Chinese population classification problems, and left 882895, 892833, 616597, 526394, 781061, and 829364 SNPs for further analyses.

Table 7 summarizes the results of our study on these sub-continental population classification problems respectively for the case of European, East Asian, African, North American, Kenyan, and Chinese population classification problems. Additional file 1: Appendix A and Figures 4, 5, 6,
7,8 , and 9 show the 10 -fold CV accuracy of the individual disjoint decision trees and ensembles of varying size built over those trees using the ETHNOPRED algorithm. The baseline accuracy calculated by simply classifying every subject to the majority class in each of these sub-continental identification problems is as follows: $61.8 \%, 54.8 \%, 40.8 \%$, $30.1 \%, 62.6 \%$, and $55.7 \%$. In each of these problems, the accuracy of a single decision tree, using 10, 12, 23, 19, 11, and 15 SNPs, is as follows: $79.0 \% \pm 5.6 \%, 74.4 \% \pm 7.9 \%, 66.2 \% \pm$ $5.3 \%, 82.7 \% \pm 5.4 \%, 79.2 \% \pm 3.5 \%$, and $47.2 \% \pm 9.1 \%$. These accuracies are significantly better than the baseline accuracy in every case except the Chinese one. Regardless of the Chinese case, ensembles of $3,39,21,11$, and 25 decision trees using $31,502,526,242$, and 271 SNPs have accuracy equal to $86.6 \% \pm 2.4 \%, 95.6 \% \pm 3.9 \%, 95.6 \% \pm$ $2.1 \%, 98.4 \% \pm 2.0 \%$, and $95.9 \% \pm 1.5 \%$ which are all statistically significantly better than the accuracy of the individual decision trees in other sub-continental classification problems. While adding additional trees to these ensembles does not improve the accuracy, using the

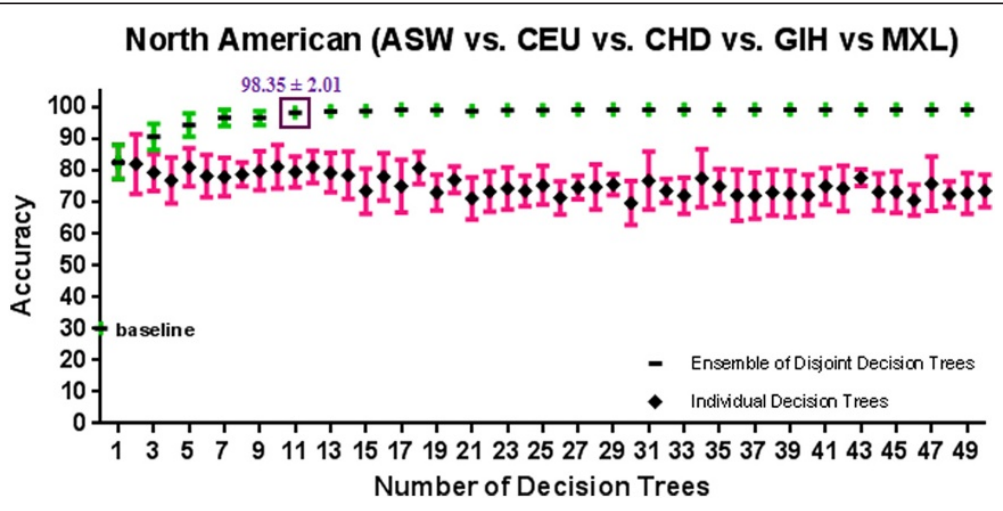

Figure 7 A comparison of 10-fold cross validation accuracy of individual decision trees and ensembles of disjoint decision trees of variable size in North American population classification problem using HapMap phase III datasets. An ensemble of 11 disjoint decision trees involving 242 SNPs has a 10 -fold cross validation accuracy of $98.3 \% \pm 2.0 \%$ which is significantly better than the baseline accuracy of $30.1 \%$. 


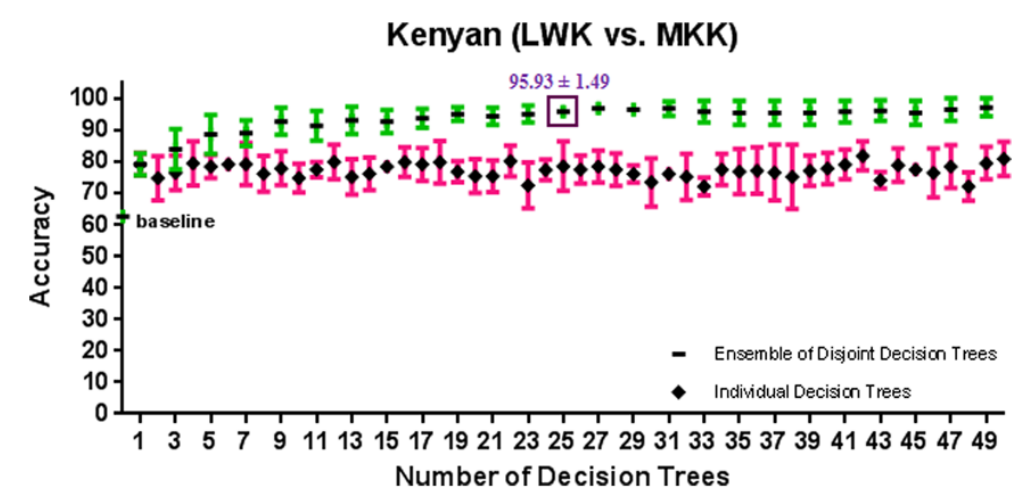

Figure $8 \mathrm{~A}$ comparison of 10-fold cross validation accuracy of individual decision trees and ensembles of disjoint decision trees of variable size in Kenyan population classification problem using HapMap phase III datasets. An ensemble of 25 disjoint decision trees involving 271 SNPs has a 10-fold cross validation accuracy of $95.9 \% \pm 1.5 \%$ which is significantly better than the baseline accuracy of $62.6 \%$.

arguments described in Predictive Modelling section, these additional trees do increase its robustness to missing SNP values; our analysis shows that an ensemble of $15,67,157$, 70, and 31 decision trees using 180, 877, 4236, 1643, and 341 SNPs guarantees both accuracy and robustness to missing values in these cases. Additional file 2: Appendix B summarizes this analysis and Additional file 4: Appendix D and Additional file 5: Appendix E show information related to the SNPs used for sub-continental population identification problems under the accuracy condition satisfaction and the robustness to missing values condition satisfaction paradigms. As mentioned above, ETHNOPRED is unable to produce a classifier that can distinguish between Chinese in Beijing and Chinese in Denver. We believe this is not a

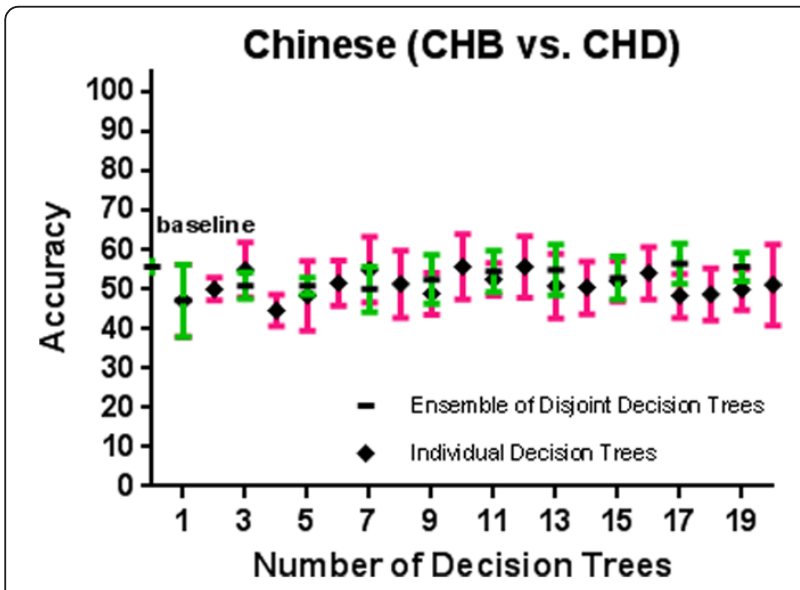

Figure 9 A comparison of 10-fold cross validation accuracy of individual decision trees and ensembles of disjoint decision trees of variable size in Chinese population classification problem using HapMap phase III datasets. We considered several individual decision trees and ensembles of various sizes, but none had 10-fold cross validation accuracy better than the baseline accuracy of $55.7 \%$. limitation of our algorithm given the fact that the first Chinese immigrant arrived in U.S. less than 200 years ago.

\section{Conclusions}

This paper presents a new algorithm called ETHNOPRED that can learn classifiers (each an ensemble of disjoint decision trees) that can identify continental and subcontinental ancestry of a person. While this task is motivated by the challenge of addressing population stratification, it might be useful in-and-of itself, to help determine a person's ancestry. Applying this approach to downstream association tests/analysis may reduce the false positive and false negative findings by (i) removing the confounding subjects or alternatively, (ii) treating population classification probabilities as a covariate. Our results show that our machine learning approach is able to find distinctions between populations when there is a distinction. Unlike AIMS, our method can accurately distinguish genetically close populations such as subgroups within Europe, East Asia, Africa, North America, and Kenya. Unlike many structured association methods, ETHNOPRED is fast and easily extendible to large scale GWASs. Furthermore, ETHNOPRED uses decision trees, which are much simpler and easier to understand than models based on principal component analysis, such as EIGENSTRAT. Note also that decision trees can be easily translated into a set of comprehensible rules, which renders the model completely transparent to the user. While EIGENSTRAT typically uses data from genome wide scans, often involving hundreds of thousands of SNPs, ETHNOPRED uses a small number of SNPs to accurately determine the ancestry of subjects. This means our method is especially useful even in the absence of whole genome (high density) SNP data (e.g., during Stage 2 or Stage 3 of a GWAS). Moreover, as it requires genotypes of only a small number of SNPs, it gets less 
affected by the genotyping errors compared with methods such as EIGENSTRAT as there is typically a smaller percentage of genotyping errors when dealing with such small number of probes. ETHNOPRED's ensemble structure makes it robust to missing values, as its multiple trees include enough redundancies that it can return accurate predictions even if it discards some decision trees while dealing with missing SNPs. We believe that this property of ETHNOPRED makes it beneficial over commonly used methods that use imputation methods for missing values, as those techniques may introduce bias or imperfect estimations. These points all argue that future GWAS studies should consider using ETHNOPRED to estimate the ethnicity of their subjects, towards addressing possible population stratification. While our ETHNOPRED system is focused on predicting ethnicity, it is within the general machine learning framework, of using training information from a group of subjects to produce a personalized classifier, that can provide useful information about subsequent subjects. This paper shows that this framework can work effectively to solve important problems.

\section{Additional files}

Additional file 1: Appendix A. 10-fold cross validation accuracy of individual decision trees and ensemble of disjoint decision trees of variable size on continental and sub-continental classification problems; in this Excel@ file, you can find the relevant accuracies for each problem on a separate sheet. In each sheet the first column specifies the decision tree index, the second column specifies the accuracy of the individual decision trees, and the third column specifies the accuracy of the ensemble of disjoint decision trees.

Additional file 2: Appendix B. Statistics for the ETHNOPRED generated classifiers accuracy and robustness to missing values metrics in different continental and sub-continental population classification problems; this Excel $^{\odot}$ file presents statistical information of each classification problem in a separate row.

Additional file 3: Appendix C. Rule-based format of the continental ancestry identification model.

Additional file 4: Appendix D. Summary statistics of SNPs used by ETHNOPRED method to tackle different continental and sub-continental population classification problems under accuracy satisfaction condition; in this Excel ${ }^{\odot}$ file, you can find the relevant summary statistics on SNPS used by our method for each problem on a separate sheet.

Additional file 5: Appendix E. Summary statistics of SNPS used by ETHNOPRED to tackle different continental and sub-continental population classification problems under robustness to missing values satisfaction condition; in this Excel ${ }^{\oplus}$ file, you can find the relevant summary statistics on SNPs used by our method for each problem on a separate sheet.

Additional file 6: Appendix F. ETHNOPRED's output file for a dataset of 696 subjects selected from a breast cancer susceptibility study in Caucasian women of Alberta, Canada [45].

Additional file 7: Appendix G. Comparison of self-declared lineage information, EIGENSTRAT's result and ETHNOPRED's result on 348 controls selected for a breast cancer susceptibility study in Caucasian women of Alberta, Canada [45].

\section{Competing interests}

The authors declare that they have no competing interests.

\section{Authors' contributions}

$\mathrm{MH}$ designed the ETHNOPRED method, conducted the experiments and drafted the manuscript; YS prepared the breast cancer dataset, performed genomic control analyses, and offered manuscript edits; JRM offered interface to clinical oncology; PR provided control samples and lineage information for breast cancer study control samples; RG participated in the experimental design and provided manuscript edits; SD conceived the plan to devise ETHNOPRED, offered the breast cancer study data, and offered manuscript edits. All authors read and approved the final manuscript.

\section{Acknowledgements}

We acknowledge financial support through the interfaculty graduate studentship from the Faculty of Medicine and Dentistry and the Faculty of Science, University of Alberta (to MH); the Natural Sciences and Engineering Research Council and the Alberta Innovates for Machine Learning (to RG); and the operating grants to projects from the Alberta Cancer Foundation and Canadian Breast Cancer Foundation- Prairies/NWT Region (to SD). The apparently healthy individuals (controls) recruited in Alberta, Canada was made possible through a provincial program, "The Tomorrow Project" funded by the Alberta Cancer Prevention and Legacy Fund, managed by Alberta Innovates-Health Solutions. We acknowledge the anonymous reviewers for their constructive suggestions and valuable feedback that improved technical quality of this paper substantially.

\section{Author details}

${ }^{1}$ Department of Computing Science, University of Alberta, Edmonton, Alberta, Canada. ${ }^{2}$ Alberta Innovates Centre for Machine Learning, University of Alberta, Edmonton, Alberta, Canada. ${ }^{3}$ Department of Laboratory Medicine and Pathology, University of Alberta, Edmonton, Alberta, Canada.

${ }^{4}$ Department of Oncology, University of Alberta, Edmonton, Alberta, Canada. ${ }^{5}$ Cancer Care, Alberta Health Services, Edmonton, Alberta, Canada. ${ }^{6}$ Department of Agricultural, Food and Nutritional Sciences, University of Alberta, Edmonton, Alberta, Canada.

Received: 28 January 2013 Accepted: 14 February 2013 Published: 22 February 2013

\section{References}

1. Jobling MA, Hurles ME, Tyler-Smith C: Human Evolutionary Genetics: Origins, Peoples and Disease. New York: Garland Science; 2004.

2. Sherry ST, Ward MH, Kholodov M, Baker J, Phan L, Smigielski EM, Sirotkin K: dbSNP: the NCBI database of genetic variation. Nucleic Acids Res 2001, 29(1):308-311.

3. Hubbard TJ, Aken BL, Beal K, Ballester B, Caccamo M, Chen Y, Clarke L, Goates G, Cunnigham F, Cutts T, Down T, Dyer SC, Fitzgerald S, FernandezBanet J, Graf S, Haider S, Hammond M, Herrero J, Holland R, Howe K, Johnson N, Kahari A, Keefe D, Kokocinski F, Kulesha E, Lawson D, Longden I, Melsopp C, Megy K, Meidl P, et al: Ensembl 2007. Nucleic Acids Res 2007, 35(Database Issue):D610-D617.

4. Lander ES, Schork NJ: Genetic dissection of complex traits. Science 1994, 265:2037-2048.

5. Hirschhorn JN, Daly MJ: Genome-wide association studies for common diseases and complex traits. Nat Rev Genet 2005, 6:95-108.

6. Freedman $M$, et al: Assessing the impact of population stratification on genetic association studies. Nat Genet 2004, 36:388-393.

7. Marchini J, et al: The effects of human population structure on large genetic association studies. Nat Genet 2004, 36:512-517.

8. Mailman MD, Feolo M, Jin Y, Kimura M, Tryka K, Bagoutdinov R, Hao L, Kiang A, Paschall J, Phan L, Popova N, Pretel S, Ziyabari L, Lee M, Shao Y, Wang ZY, Sirotkin K, Ward M, Kholodov M, Zbicz K, Beck J, Kimelman M, Shevelev S, Preuss D, Yaschenko E, Graeff A, Ostell J, Sherry ST: The NCBI $\mathrm{dbGaP}$ database of genotypes and phenotypes. Nat Genet 2007, 39(10):1181-1186.

9. Hindorff LA, Sethupathy P, Junkins HA, Ramos EM, Mehta JP, Collins FS, Manolio TA: Potential etiologic and functional implications of genomewide association loci for human diseases and traits. P Natl Acad Sci 2009, 106(23):9362-9367.

10. Cardon LR, Palmer LJ: Population stratification and spurious allelic association. Lancet 2003, 361:598-604. 
11. Wu C, DeWan A, Hoh J, Wang Z: A comparison of association methods correcting for population stratification in case-control studies. Ann Hum Genet 2011, 75(3):418-427.

12. Enoch MA, Shen PH, Xu K, Hodgkinson C, Goldman D: Using ancestryinformative markers to define populations and detect population stratification. J Psychopharmacol 2006, 20(4 Suppl):19-26.

13. Kosoy R, Nassir R, Tian C, White PA, Butler LM, Silva G, Kittles R, AlarconRiquelme ME, Gregersen PK, Belmont JW, et al: Ancestry informative marker sets for determining continental origin and admixture proportions in common populations in America. Hum Mutat 2009 30(1):69-78

14. Nassir R, Kosoy R, Tian C, White PA, Butler LM, Silva G, Kittles R, AlarconRiquelme ME, Gregersen PK, Belmont JW, De La Vega FM, Seldin MF: An ancestry informative marker set for determining continental origin: validation and extension using human genome diversity panels. BMC Genet 2009, 10:39.

15. Phillips C, Salas A, Sanchez JJ, Fondevila M, Gomez-Tato A, Alvarez-Dios J, Calaza M, de Cal MC, Ballard D, Lareu MV, et al: Inferring ancestral origin using a single multiplex assay of ancestry-informative marker SNPs. Forensic Sci Int Genet 2007, 1(3-4):273-280.

16. Halder I, Shriver M, Thomas M, Fernandez JR, Frudakis T: A panel of ancestry informative markers for estimating individual biogeographical ancestry and admixture from four continents: utility and applications. Hum Mutat 2008, 29(5):648-658.

17. Campbell CD, Ogburn EL, Lunetta KL, Lyon HN, Freedman ML, Groop LC, Altshuler D, Ardlie KG, Hirschhorn JN: Demonstrating stratification in a European American population. Nat Genet 2005, 37(8):868-872.

18. Seldin MF, Shigeta R, Villoslada P, Selmi C, Tuomilehto J, Silva G, Belmont JW, Klareskog L, Gregersen PK: European population substructure: clustering of northern and southern populations. PLoS Genet 2006, 2(9):e143.

19. Helgason A, Yngvadottir B, Hrafnkelsson B, Gulcher J, Stefansson K: An Icelandic example of the impact of population structure on association studies. Nat Genet 2005, 37(1):90-95.

20. Seldin MF, Price AL: Application of ancestry informative markers to association studies in European Americans. PLoS Genet 2008, 4(1):e5.

21. Tian C, Plenge RM, Ransom M, Lee A, Villoslada P, Selmi C, Klareskog L, Pulver AE, Qi L, Gregersen PK, et al: Analysis and application of European genetic substructure using $300 \mathrm{~K}$ SNP information. PLoS Genet 2008, 4(1):e4

22. Tian C, Kosoy R, Lee A, Ransom M, Belmont JW, Gregersen PK, Seldin MF: Analysis of East Asia genetic substructure using genome-wide SNP arrays. PLoS One 2008, 3(12):e3862.

23. Bryc K, Auton A, Nelson MR, Oksenberg JR, Hauser SL, Williams S, Froment A, Bodo JM, Wambebe C, Tishkoff SA, Bustamante CD: Genomewide patterns of population structure and admixture in West Africans and African Americans. PNAS 2010, 107:786-791.

24. Tian C, Hinds DA, Shigeta R, Adler SG, Lee A, Pahl MV, Silva G, Belmont JW, Hanson RL, Knowler WC, et al: A genomewide single-nucleotide -polymorphism panel for Mexican American admixture mapping. Am J Hum Genet 2007, 80(6):1014-1023.

25. Bauchet M, McEvoy B, Pearson LN, Quillen EE, Sarkisian T, Hovhannesyan K, Deka R, Bradley DG, Shriver MD: Measuring European population stratification with microarray genotype data. Am J Hum Genet 2007, 80(5):948-956.

26. Devlin B, Roeder K: Genomic control for association studies. Biometrics 1999, 55:997-1004

27. Reich D, Goldstein D: Detecting association in a case-control study while allowing for population stratification. Genet Epidemiol 2001, 20:4-16.

28. Devlin B, et al: Genomic control to the extreme. Nat Genet 2004, 36:1129-1130.

29. Clayton DG, et al: Population structure, differential bias and genomic control in a large-scale, case-control association study. Nat Genet 2005, 37:1243-1246.

30. Pritchard JK, Stephens M, Rosenberg NA, Donnelly P: Association mapping in structured populations. Am J Hum Genet 2000, 67:170-181.

31. Satten $G$, et al: Accounting for unmeasured population substructure in case-control studies of genetic association using a novel latent-class model. Am J Hum Genet 2001, 68:466-477.

32. Pritchard $J K$, et al: Inference of population structure using multilocus genotype data. Genetics 2000, 155:945-959.

33. Rosenberg NA, et al: Genetic structure of human populations. Science 2002, 298:2381-2385.
34. Price $\mathrm{AL}$, et al: Principal components analysis corrects for stratification in genome-wide association studies. Nat Genet 2006, 38:904-909.

35. Patterson N, Price AL, Reich D: Population structure and eigenanalysis. PLoS Genet 2006, 2:e190.

36. Novembre J, Stephens M: Interpreting principal component analyses of spatial population genetic variation. Nat Genet 2008, 40:646-649.

37. McCarthy MI, Abecasis GR, Cardon LR, Goldstein DB, Little J, loannidis JP: Genome-wide association studies for complex traits: consensus, uncertainty and challenges. Nat Rev Genet 2008, 9:356-369.

38. Ahn K, Gordon D, Finch SJ: Increase of rejection rate in case-control studies with the differential genotyping error rates. Stat Appl Genet Mol Biol 2009, 8(1). Article25.

39. Clayton DG, Walker NM, Smyth DJ, Pask R, Cooper JD, Maier LM, Smink LJ, Lam AC, Ovington NR, Stevens HE, et al: Population structure, differential bias and genomic control in a large-scale, case-control association study. Nat Genet 2005, 37(11):1243-1246.

40. Kang SJ, Finch SJ, Haynes C, Gordon D: Quantifying the percent increase in minimum sample size for SNP genotyping errors in genetic modelbased association studies. Hum Hered 2004, 58(3-4):139-144.

41. Londono D, Haynes C, De La Vega FM, Finch SJ, Gordon D: A cost-effective statistical method to correct for differential genotype misclassification when performing case-control genetic association. Hum Hered 2010 70(2):102-108

42. Moskvina V, Craddock N, Holmans P, Owen MJ, O'Donovan MC: Effects of differential genotyping error rate on the type I error probability of casecontrol studies. Hum Hered 2006, 61(1):55-64.

43. Plagnol V, Cooper JD, Todd JA, Clayton DG: A method to address differential bias in genotyping in large-scale association studies. PLoS Genet 2007, 3(5):e74.

44. Rice KM, Holmans P: Allowing for genotyping error in analysis of unmatched case-control studies. Ann Hum Genet 2003, 67(Pt 2):165-174.

45. Rakovski CS, Stram DO: A kinship-based modification of the armitage trend test to address hidden population structure and small differential genotyping errors. PLoS One 2009, 4(6):e5825.

46. The International HapMap Consortium: The International HapMap Project. Nature 2003, 426:89-796

47. Sehrawat B, Sridharan M, Ghosh S, Robson P, Cass CE, Mackey J, Greiner R, Damaraju S: Potential novel candidate polymorphisms identified in genome-wide association study for breast cancer susceptibility. Hum Genet 2011, 130(4):529-37.

48. Pearson K: Mathematical contributions to the theory of evolution. XI. On the influence of natural selection on the variability and correlation of organs. Philos Trans R Soc Lond 1903, Ser A, 200(321-330):1-66.

49. Mitchell T: Machine Learning. New York: McGraw Hill; 1997.

50. Duda RO, Hart PE, Stork DG: Pattern classification. 2nd edition. New York: Wiley; 2001.

51. Hastie T, Tibshirani R, Friedman J: The Elements of Statistical Learning: Data Mining, Inference, and Prediction. 2nd edition. New York: Springer; 2009.

52. Baldi P, Brunak S: Bioinformatics: The Machine Learning Approach. 2nd edition. Cambridge, Massachusetts: The MIT Press; 2001.

53. Larranaga P, Calvo B, Santana R, Bielza C, Galdiano J, Inza I, Lozano JA, Armananzas R, Santafe G, Perez A, Robles A: Machine learning in bioinformatics. Brief Bioinform 2006, 7(1):86-112.

54. Tarca AL, Carey VJ, Chen XW, Romero R, Draghici S: Machine learning and its applications to biology. PLoS Comput Biol 2007, 3(6):e116.

55. Mathé C, Sagot M-F, Schiex T, Rouzé P: Current methods of gene prediction, their strengths and weaknesses. Nucleic Acids Res 2002, 30:4103-4117

56. Won K, Prugel-Bennett A, Krogh A: Training HMM structure with genetic algorithm for biological sequence analysis. Bioinformatics 2004, 20(18):3613-3619.

57. Yi TM, Lander ES: Protein secondary structure prediction using nearestneighbor methods. J Mol Biology 1993, 232:1117-1129.

58. Pirooznia M, Yang JY, Yang MQ, Deng Y: A comparative study of different machine learning methods on microarray gene expression data. BMC Genomics 2008, 9(Suppl 1):S13.

59. Middendorf M, Kundaje A, Wiggins C, Freund Y, Leslie C: Predicting genetic regulatory response using classification. Bioinformatics 2004 20(Suppl 1):|232-1240. 
60. Zhou GD, Shen D, Zhang J, Su J, Tan SH: Recognition of protein/gene names from text using an ensemble of classifiers. BMC Bioinformatics 2005, 6(Suppl 1):S7.

61. Quinlan JR: Induction of decision trees. Mach Learn 1986, 1:81-106.

62. Breiman L, Friedman JH, Olshen RA, Stone CJ: Classification and Regression Trees. New York: Chapman \&Hall (Wadsworth, Inc.); 1984.

63. Dietterich TG: Ensemble methods in machine learning. Lect Notes Comput Sc 2000, 1857:1-15.

64. Kuncheva LI, Whitaker CJ: Measures of diversity in classifier ensembles and their relationship with the ensemble accuracy. J Mach Learn 2003, 51(2):181-207.

65. Tan AC, Gilbert D: Ensemble machine learning on gene expression data for cancer classification. Appl Bioinformatics 2003, 2:S75-\$83.

66. Peng Y: A novel ensemble machine learning for robust microarray data classification. Comput Biol Med 2006, 36(6):553-573.

67. Polikar R: Ensemble based systems in decision making. IEEE Circuits Syst Mag 2006, 6(3):21-45.

68. Dudoit S, Fridlyand J, Speed TP: Comparison of discrimination methods for the classification of tumors using gene expression data. J Am Stat Assoc 2002, 97(457):77-87.

69. Lin DY, Hu Y, Huang BE: Simple and efficient analysis of disease association with missing genotype data. Am J Hum Genet 2008, 82(2):444-452.

70. Boulesteix AL, Strobl C, Augustin T, Daumer M: Evaluating microarraybased classifiers: an overview. Cancer Informatics 2008, 6:77-97.

Cite this article as: Hajiloo et al:: ETHNOPRED: a novel machine learning method for accurate continental and sub-continental ancestry identification and population stratification correction. BMC Bioinformatics $201314: 61$.

\section{Submit your next manuscript to BioMed Central and take full advantage of:}

- Convenient online submission

- Thorough peer review

- No space constraints or color figure charges

- Immediate publication on acceptance

- Inclusion in PubMed, CAS, Scopus and Google Scholar

- Research which is freely available for redistribution 